\title{
PET / CT Imaging in Fever of Unknown Origin: A Case of Relapsing Polychondritis
}

HONG-LEI LIU, MD, Department of Rheumatology and Immunology, Rui'jin Hospital, School of Medicine, Shanghai Jiao'tong University, Shanghai, China; CHENG-DE YANG, MD, Department of Rheumatology and Immunology, Rui'jin Hospital, School of Medicine, Shanghai Jiao'tong University, Shanghai, China. Supported by grants from the National Natural Science Foundation of China (number 81502016). Address correspondence to Dr. C.D. Yang, Department of Rheumatology and Immunology, Rui'jin Hospital, School of Medicine, Shanghai Jiao'tong University, No. 197 Ruijin Second Road, Huang'pu District, Shanghai 200025, People's Republic of China. E-mail: yangchengde@sina.com. J Rheumatol 2017;44:1098; doi:10.3899/jrheum.160986. Written consent to publish was obtained. Ethics board approval with 2016(62) was attained from the Rui'jin Hospital Ethics Committee.

Relapsing polychondritis is a rare disease, and the cases characterized only by fever and restricted airway involvement are extremely rare.

A 44-year-old febrile man without any other symptoms and signs was admitted to Rui'jin Hospital for further investigation, where $18 \mathrm{~F}$-fluorodeoxyglucose positron emission tomography/computed tomography (FDG-PET/CT) showed an 18FDG accumulation and indicated local acute inflammation in the trachea and bronchus. Other routine tests for infections, autoimmunological antibodies, and bone marrow biopsy were normal.

Erythrocyte sedimentation rate was $102 \mathrm{~mm} / \mathrm{h}$ (normal range $0-20 \mathrm{~mm} / \mathrm{h}$ ) and C-reactive protein level was $97 \mathrm{mg} / \mathrm{l}$ (normal range $0-10 \mathrm{mg} / \mathrm{l}$ ). Anticollagen type II antibody was raised to $97 \mathrm{EU} / \mathrm{ml}$ (normal $<20 \mathrm{EU} / \mathrm{ml}$ ). Chest CT showed the circumferential thickening and narrowing of tracheal walls or chondromalacia. PET/CT showed a diffuse FDG accumulation with a SUVmax 4.6 in the broadly thickened trachea and bronchus (Figure 1) with no involvement of the ears.

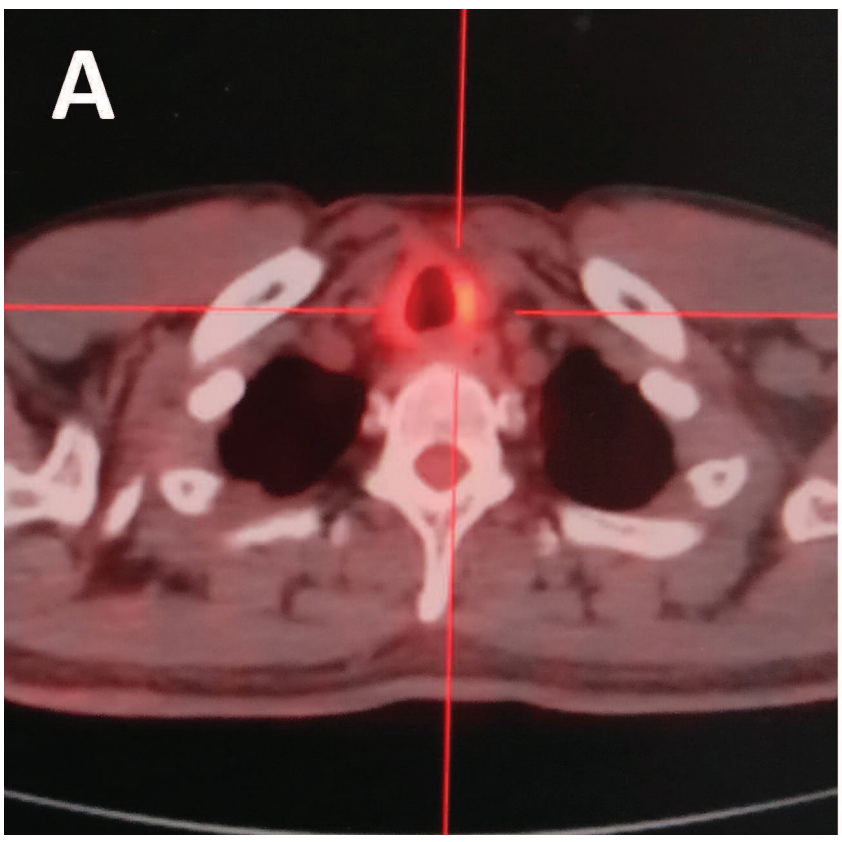

Relapsing polychondritis has a poor prognosis because it tends to present with obstructive respiratory failure, most frequently expiratory collapse of the tracheobronchial tree. Evaluation of relapsing polychondritis can be complex and should include a variety of diagnostic techniques. PET/CT is an alternative to invasive methods for examination for local inflammation of the involved organs and tissues in patients with relapsing polychondritis $1,2,3,4$.

\section{REFERENCES}

1. Suzuki S, Ikegami A, Hirota Y, Ikusaka M. Fever and cough without pulmonary abnormalities on CT: relapsing polychondritis restricted to the airways. Lancet 2015;385:88.

2. De Geeter F, Vandecasteele SJ. Fluorodeoxyglucose PET in relapsing polychondritis. N Engl J Med 2008;358:536-7.

3. Cassone G, Lo Gullo A, Bajocchi G, Salvarani C. [18F]fluorodeoxyglucose positron emission tomography imaging in a case of relapsing polychondritis. Rheumatology 2012;51:1813.

4. Zhou H, Su M, Li L. 18F-FDG PET/CT imaging of relapsing polychondritis: a case report. Medicine 2016;95:e4496.

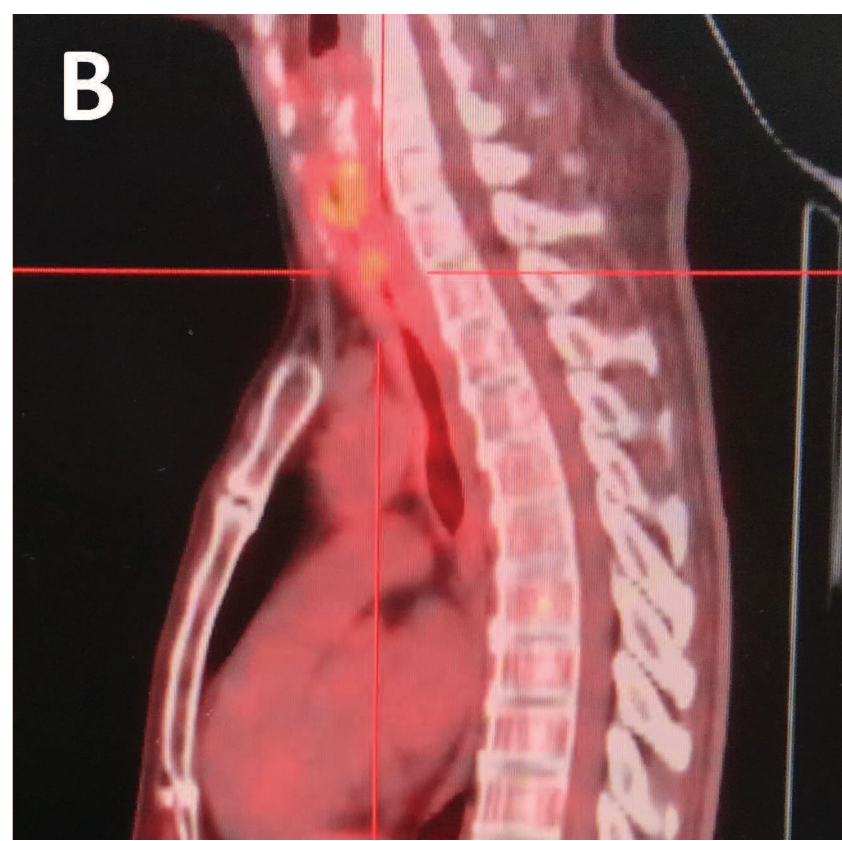

Figure 1. Relapsing polychondritis restricted to airway. A diffuse $18 \mathrm{~F}$-fluorodeoxyglucose accumulation in thickened trachea and bronchus on positron emission tomography/computed tomography. (A) Cross-section view. (B) Vertical plane view. 